\title{
A General Method for Precalculation of Parameters for Sustained Off Resonance Irradiation/Collision-Induced Dissociation
}

\author{
Ekaterina Mirgorodskaya, Peter B. O'Connor, and Catherine E. Costello \\ Mass Spectrometry Resource, Department of Biochemistry, Boston University School of Medicine, Boston,
} Massachusetts, USA

\begin{abstract}
Sustained off resonance irradiation (SORI) collision-induced dissociation (CID) is a commonly used method of collisionally activating ions for fragmentation in Fourier transform mass spectrometric experiments. To achieve the degree of fragmentation desired, both the irradiation frequency and amplitude must be optimized. This is a time-consuming procedure, particularly when the $\mathrm{m} / \mathrm{z}$ values of the precursor ions vary over a broad range. We present an approach that simplifies this optimization by precalculating the irradiation frequency of the ions to be fragmented as a constant percentage of the reduced cyclotron frequency. Using this approach, the optimal amplitude was found to be significantly less dependent on the $\mathrm{m} / \mathrm{z}$ value of the precursor ion, and therefore required little or no adjustment. This method considerably simplified optimization of SORI-CID for analysis of carbohydrates, glycoconjugates, and peptides over the mass range $\mathrm{m} / \mathrm{z} 300-3500$, requiring optimization of only a single experimental parameter, the irradiation amplitude, and only for the first MS/MS stage. (J Am Soc Mass Spectrom 2002, 13, 318-324) (C) 2002 American Society for Mass Spectrometry
\end{abstract}

$\mathrm{T}$ The high resolution and mass accuracy achievable with a Fourier transform mass spectrometer (FTMS) [1-3], in combination with the high ionization efficiency of matrix-assisted laser desorption/ ionization (MALDI) [4], portend wide application to a broad range of biological samples. The capability for multistage dissociation of MALDI-generated ions and the choice of activation techniques available with the FT mass analyzer provide the opportunity to obtain detailed structural information on the analyzed compounds. SORI-CID [5] is a general method of collisionally activating ions for fragmentation by irradiating them with a single off-resonant sine wave excitation pulse while the background gas pressure is raised to the $10^{-6}$ torr region. The resulting large number of sequential low-energy collisions slowly heats the activated ions so that dissociation mainly occurs through the lowest energy fragmentation channels. SORI-CID has, therefore, been extensively used for structural analysis of various biopolymers such as proteins and polypeptides [6-19], carbohydrates [20-24], oligonucleotides $[25,26]$, and polymers [27].

Four parameters determine the fragmentation efficiency of SORI-CID: The SORI pulse irradiation frequency, amplitude and duration, and the pressure of

Published online February 13, 2002

Address reprint requests to Dr. C. E. Costello, Mass Spectrometry Resource, Boston University School of Medicine, 715 Albany Street, R-806, Boston, MA 02118-2526, USA. E-mail: cecmsms@bu.edu the collision gas $[28,29]$. The SORI frequency and amplitude are coupled as they together control the lab-frame collision energy of the ions, and are typically optimized for each individual SORI-CID event to obtain the desired degree of fragmentation. When SORI-CID is applied to ions within a narrow $\mathrm{m} / \mathrm{z}$ range, the optimization can be performed by slight adjustment of the irradiation amplitude while maintaining a constant frequency offset $(\Delta \nu)$ between the reduced cyclotron frequency $\left(\nu_{+}\right)$and the SORI frequency $\left(\nu_{\text {SORI }}\right)$. Thus, for electrospray-generated ions of proteins and peptides, a $\Delta \nu$ of $1-2 \mathrm{kHz}$ has been reported optimal to minimize isotopic distortion as well as the width of the blind spot, i.e., the frequency range where resonant ion excitation causes ion ejection [9]. Since MALDI generates predominantly singly-charged ions, the $\mathrm{m} / \mathrm{z}$ range of the ions is relatively broad compared to that observed for electrospray-generated multiply-charged ions. If $\Delta \nu$ is kept constant for ions varying over a broad frequency range $(\mathrm{m} / \mathrm{z}$ range), the optimal amplitude varies greatly. Consequently, optimization of the SORICID parameters can be particularly time-consuming for MALDI experiments.

In the current paper, we propose a simple way to optimize the parameters for SORI-CID of glycoconjugates and polypeptides. For all ions selected for fragmentation, the irradiation frequency offset is set to $1.5 \%$ of the reduced cyclotron frequency, i.e., $\Delta \nu=0.015 \nu_{+}$. With this approach, the optimal amplitude was found to be significantly less dependent on the $\mathrm{m} / \mathrm{z}$ value of 
the precursor ion, and therefore required little or no adjustment. This was investigated for ions of $m / z$ varying over an order of magnitude $(\mathrm{m} / \mathrm{z} 300-3500)$. In many cases, the irradiation amplitude needed only to be fine-tuned to influence the relative abundances of specific fragment ions. Furthermore, for oligosaccharide and glycolipid samples, which often require several dissociation stages for their detailed structural analysis, the optimal amplitude of the SORI-CID event was found to be stable through each stage and independent of the $m / z$ of the selected precursor ions. The method is particularly valuable for MALDI SORI-CID experiments, but is also useful for SORI-CID of ESI-generated ions.

\section{Experimental}

\section{Materials}

MALDI matrices, 6-aza-2-thiothymine (ATT) and 2,5dihydroxybenzoic acid (DHB), were purchased from Aldrich (Aldrich Chemical Co., Milwaukee, WI). The carbohydrate sample, obtained from a parasite lipophosphoglycan by mild acid hydrolysis, was provided by B. N. Singh (SUNY Health Science Center, Syracuse, NY) as part of an on-going collaboration. The glycolipid sample, galactosylceramide from pig intestine, was a gift from S. Teneberg and K.-A. Karlsson (Göteborg University, Sweden). The peptides, D-Ala ${ }^{2}$ Met $^{5}$-enkephalinamide $\left(\mathrm{YAGFM}-\mathrm{NH}_{2}\right),[\mathrm{M}+\mathrm{H}]^{+} \mathrm{m} / \mathrm{z}$ 587.3; $\mathrm{Arg}^{8}$-vasopressin (CYFQNCPRG- $\mathrm{NH}_{2}$ ), $[\mathrm{M}+\mathrm{H}]^{+} \mathrm{m} / z$ 1086.5; fibrinopeptide A (ADSGEGDFLAEGGGVR$\mathrm{OH}),[\mathrm{M}+\mathrm{H}]^{+} \mathrm{m} / z$ 1537.7; and oxidized insulin $\beta$-chain (FVNQHLC $_{\text {ox }}$ GSHLVEALYLVC $_{\text {ox }}$ GERGFFYTPKA-OH), $[\mathrm{M}+\mathrm{H}]^{+} \mathrm{m} / \mathrm{z}$ 3494.7, were purchased from Sigma (Sigma, St. Louis. MO). All masses listed here and throughout the paper are the mass of the monoisotopic peak.

\section{MALDI FTMS}

The experiments were performed on an Ultima FTMS (IonSpec, Irvine, CA) equipped with a $7 \mathrm{~T}$ actively shielded superconducting electromagnet (Cryomagnetics, Oak Ridge, TN). The instrument has two separate vacuum systems. One vacuum system is equipped with an Analytica electrospray ion source (Branford, CT); the other with an external IonSpec MALDI ion source. The MALDI source uses a 10-faceted sample probe and a $337 \mathrm{~nm}$ nitrogen laser (Laser Sciences, Franklin, MA) with a motor-driven beam steering mirror (Newport Optics, Irvine, CA), a motor-driven focusing lens (Newport Optics, Irvine, CA), an iris, and a gradient filter for manually adjusting the laser power. Ions are transferred via a quadrupole ion guide to a capacitively coupled closed cylindrical cell similar to the design by Beu et al. [30], but with additional external trapping plates.

Saturated solutions of the matrix compounds ATT and DHB in methanol were used for sample prepara- tion. Carbohydrate and glycolipid samples were prepared using ATT as the matrix. The ATT matrix solution was deposited onto the probe tip using Eppendorf GELoader pipette tips (Brinkmann Instruments Inc., Westbury, NY) resulting in microcrystalline layers of matrix, followed by sample deposition onto the preformed matrix layer. For carbohydrates, $0.6 \mu \mathrm{L}$ of the sample dissolved in water were deposited onto the matrix layer. For glycolipid samples, $1 \mu \mathrm{L}$ of $20 \mathrm{mM}$ lithium acetate (LiAc) was placed on the matrix layer prior to sample deposition in order to enhance the formation of lithiated molecular ions. Glycolipid samples $(10 \mathrm{pmol} / \mu \mathrm{L})$ in chloroform:methanol (2:1, vol/ vol), were deposited successively, in small portions onto the preformed matrix layers using Eppendorf GELoader pipette tips to prevent complete dissolvation of the matrix layer. Polypeptide samples were dissolved in water $(10-20 \mathrm{pmol} / \mu \mathrm{L})$, and were prepared using both ATT and DHB as MALDI matrices. With ATT, peptide samples were prepared in the same way as carbohydrate samples. With DHB, $0.6 \mu \mathrm{L}$ of the peptide sample was placed on the probe tip followed by addition of $0.3 \mu \mathrm{L}$ of $\mathrm{DHB}$ solution.

\section{SORI-CID Analysis}

Ions of interest were isolated using the arbitrary waveform generator [31], with parameters adjusted to eject unwanted ions with minimal loss of the intended precursor ion. The duration of the SORI pulse and collision gas pressure were fixed for all experiments, leaving the off-resonance irradiation frequency and the amplitude of the SORI pulse as the two adjustable parameters to control the degree of fragmentation. SORI-CID was performed using pulsed introduction of $\mathrm{N}_{2}$ collision gas. The pulse valve was opened for $4 \mathrm{~ms}$, $100 \mathrm{~ms}$ prior to the SORI event in order to transiently increase the pressure in the analyzer region to $10^{-6}$ torr. In the following $2 \mathrm{~s}$, the background pressure dropped to low $10^{-9}$ torr as the cell was evacuated. The SORI pulse duration was $2 \mathrm{~s}$ with the irradiation frequency offset set to $1.5 \%$ of the resonant frequency of the selected precursor ion and the initial amplitude held constant at $10 \mathrm{~V}_{\mathrm{bp}}$. Depending on the type of the analyzed compound and the desired degree of fragmentation, the amplitude of the SORI pulse was further optimized. A delay of $6-8 \mathrm{~s}$ preceded the ion detection event to allow the pressure to return into the low $10^{-10}$ torr region.

All SORI-CID experiments were performed using a pulsed valve to transiently increase the pressure to $\sim 10^{-6}$ torr. Under these conditions, the collision gas pressure, which is one of the parameters influencing SORI-CID fragmentation efficiency [28, 29], is not stable through the SORI-CID event. However, for SORI-CID, pulsed gas introduction has a number of advantages over a static pressure experiment. First, all commercially available FTMS instruments are equipped with a pulse valve, while high quality leak valve and ballast- 
ing systems are less common. Second, it requires a shorter pump-down delay time prior to detection. A set of SORI-CID experiments, using fibrinopeptide A as a model compound, was performed to determine that there was no loss in fragmentation efficiency using pulsed gas introduction in comparison to maintaining static pressures. Since we currently do not have a leak-valve for maintaining a constant pressure in the ICR cell, we rapidly fired the pulse valve to maintain elevated pressure in the $10^{-6}-10^{-7}$ torr range during the SORI-CID event. For collision gas pressure in $10^{-6}$ torr region, there was no noticeable difference in the type of the generated fragment ions nor in the fragmentation efficiency when the results from single-pulse or multiple-pulse experiments were compared. Most likely, pulsed gas introduction allows the pressure to sweep through the range of pressure regimes, ensuring that all ions experience optimal pressure [29] for efficient fragmentation during SORI activation. Therefore, collision gas pressure was not used as a variable parameter for SORI-CID optimization, but was kept the same for all SORI-CID experiments and was transiently increased using a single pulsed valve event into the $10^{-6}$ torr region.

\section{Results}

Oligosaccharides and glycolipids are readily detected as cationized molecular ions in MALDI FTMS experiments $[32,33]$. Due to the structural heterogeneity of glycans and glycoconjugates in biological systems, samples purified for MS analysis frequently represent complex mixtures, with components varying in molecular weight by a few mass units to several hundred. For each sample component, the structural characterization often requires several decomposition stages. Because the $\mathrm{m} / \mathrm{z}$ values of the precursor ions vary widely through the sequential MS/MS stages, optimization of the SORICID parameters for each sample component and each MS/MS stage is a time consuming procedure. To simplify this procedure, the irradiation frequency offset was calculated as a percentage of the reduced cyclotron frequency. As a result, the irradiation amplitude required little or no optimization. An example of this approach is shown in Figure 1 for a 2-aminobenzamide $(2 \mathrm{AB})$ derivative of an unknown oligosaccharide obtained from a parasite lipophosphoglycan by mild acid hydrolysis. The sodiated molecular ion at $\mathrm{m} / \mathrm{z} 1240.5$ was subjected to SORI-CID analysis (Figure 1a). The irradiation frequency offset $(\Delta \nu)$ was set to $1.5 \%$ of the reduced cyclotron frequency $\left(\nu_{+}\right)$of the precursor ion $\left(\Delta \nu=0.015 \nu_{+}\right)$. Irradiation amplitude of $10 \mathrm{~V}_{\mathrm{bp}}$ was found optimal, yielding extensive fragmentation with fragment ions over a broad mass range. The observed fragment ions indicated the presence of two HexNAc residues at the non-reducing terminus and a deoxyHex residue at the reducing terminus, followed by a Hex residue. To confirm this assignment and to complete the determination of the oligosaccharide sequence, selected
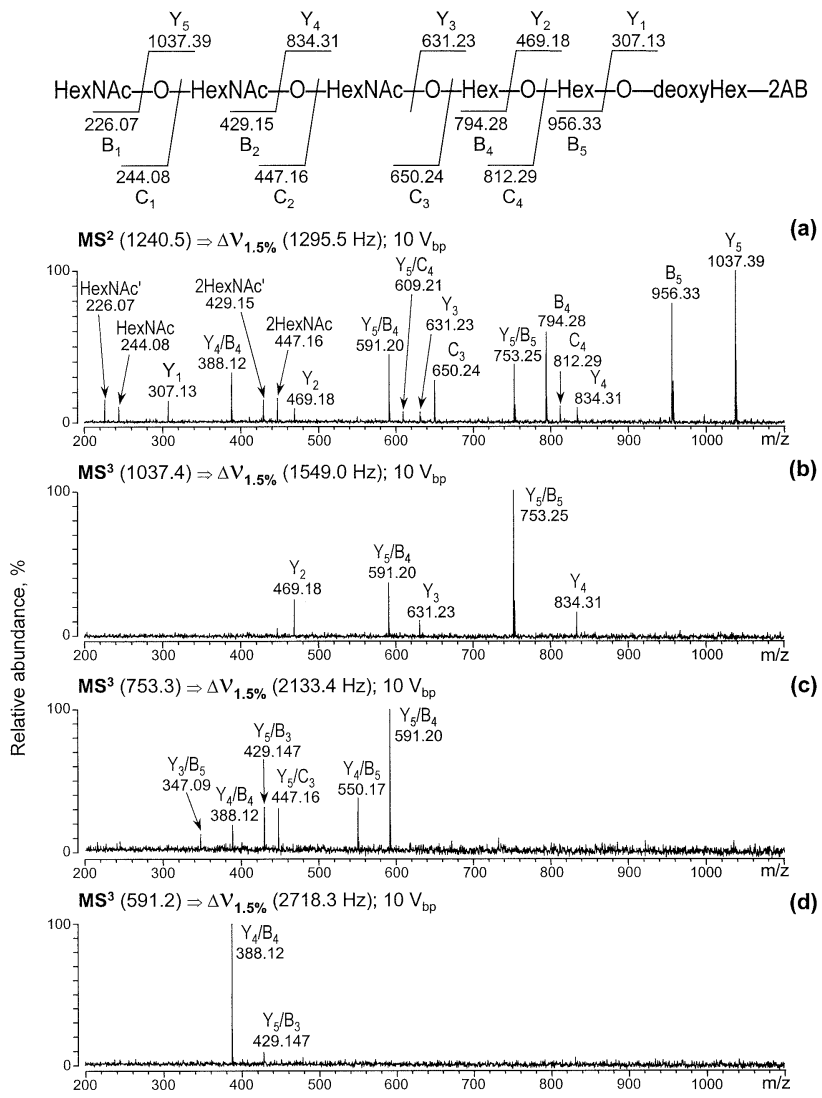

Figure 1. MALDI FT SORI-CID mass spectra of the 2-aminobenzamide (2AB) derivative of an unknown oligosaccharide: (a) $\mathrm{MS}^{2}$ of the $m / z 1240.5$ using $\Delta \nu=1.5 \% \nu_{+}(1295.5 \mathrm{~Hz}) ;(\mathbf{b}) \mathrm{MS}^{3}$ of the $m / z 1037.4(\Delta \nu=1549.0 \mathrm{~Hz})$; (c) $\mathrm{MS}^{3}$ of the $\mathrm{m} / \mathrm{z} 753.3(\Delta \nu=2133.4$ $\mathrm{Hz}) ;(\mathbf{d}) \mathrm{MS}^{3}$ of the $m / z 591.2(\Delta \nu=2718.3 \mathrm{~Hz})$. The amplitude of the RF burst was $10 \mathrm{~V}_{\mathrm{bp}}$ for all the experiments. All ions were observed as sodiated species. Fragments are labeled according to the Domon and Costello nomenclature [38]. The prime symbol (') indicates a dehydrated analog of a given ion.

primary fragment ions were isolated and subjected to further collisional activation (Figure $1 \mathrm{~b}-\mathrm{d}$ ). For each isolated ion, $\Delta \nu$ was set to $1.5 \%$ of $\nu_{+}$, while the irradiation amplitude was held constant at $10 \mathrm{~V}_{\mathrm{bp}}$. These settings yielded good results for each of the $\mathrm{MS}^{3}$ experiments, and no significant improvement was observed by manual tuning of the SORI amplitude. The observed fragments resulting from glycosidic bond cleavages identified the oligosaccharide as $\mathrm{HexNAc}_{3}-$ $\mathrm{Hex}_{2}$ deoxyHex-2AB (Figure 1). In this example, $\Delta \nu$ varied from $1295 \mathrm{~Hz}$ for $\mathrm{m} / \mathrm{z} 1240.5$ (Figure 1a) to 2718 $\mathrm{Hz}$ for $\mathrm{m} / \mathrm{z} 591.2$ (Figure 1d), and the optimal amplitude was found to remain constant through all SORI-CID experiments. This observation was consistent for other carbohydrate samples from different sources. Thus, the calculation of $\Delta \nu$ as a constant percentage of $\nu_{+}$simplified the SORI-CID analysis of carbohydrate samples by requiring optimization of only a single parameter: the SORI amplitude for the first MS/MS stage.

A glycoconjugate class currently studied in our laboratory is glycosphingolipids (GSLs). GSLs consist of a hydrophilic head group (the carbohydrate moiety) and 


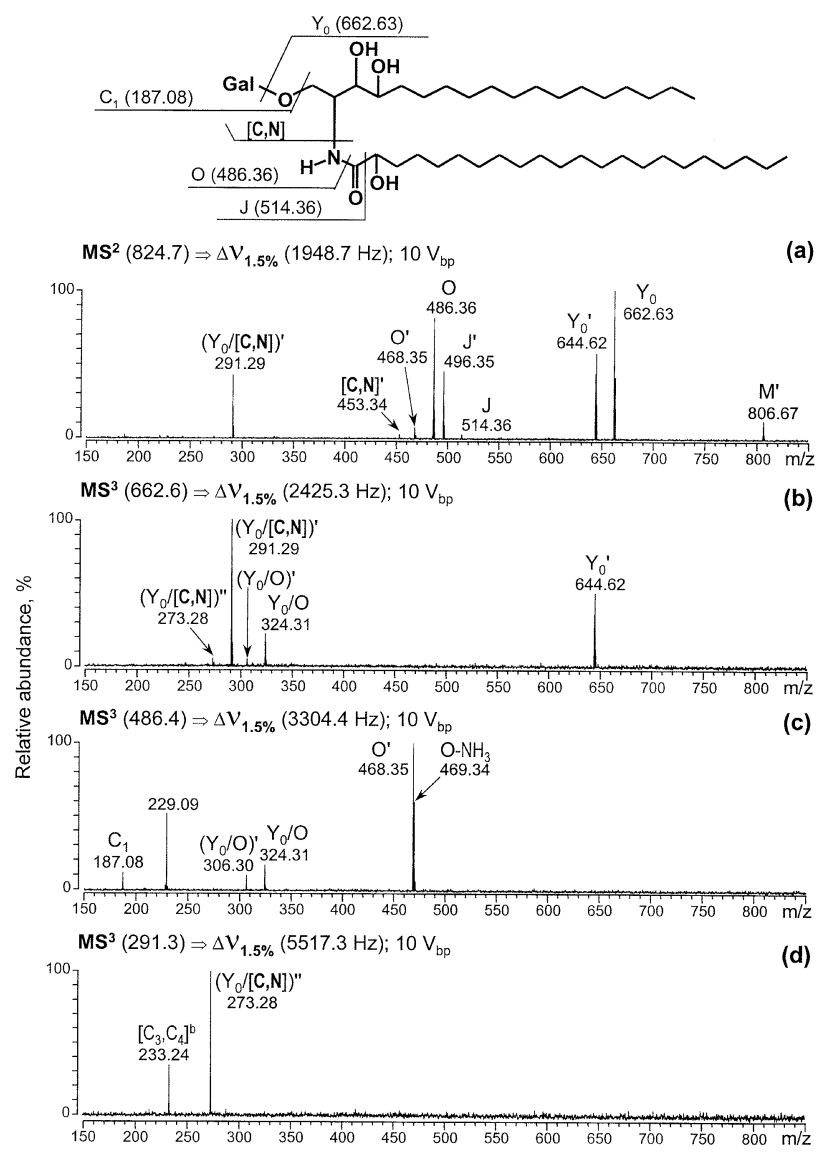

Figure 2. MALDI FT SORI-CID mass spectra of a glycosphingolipid using $\Delta \nu=1.5 \% \nu_{+}:$(a) $\mathrm{MS}^{2}$ of the $[\mathrm{M}+\mathrm{Li}]^{+}$at $m / z 824.68$ $(\Delta \nu=1948.5 \mathrm{~Hz}) ;(\mathbf{b}) \mathrm{MS}^{3}$ of $\mathrm{m} / \mathrm{z} 662.6(\Delta \nu=2425.3 \mathrm{~Hz}) ;(\mathbf{c}) \mathrm{MS}^{3}$ of $\mathrm{m} / \mathrm{z} 486.4(\Delta \nu=3304.4 \mathrm{~Hz})$; (d) $\mathrm{MS}^{3}$ of $\mathrm{m} / z 291.3(\Delta \nu=5517.3$ $\mathrm{Hz}$ ). The amplitude of the RF burst was $10 \mathrm{~V}_{\mathrm{bp}}$ for all the experiments. All ions were observed as lithiated species. Fragments are labeled according to Domon and Costello nomenclature [38], as modified by Perreault and Costello [39] and Adams and Ann [34]. The prime symbol (') indicates a dehydrated analog of a given ion. All mass values represent the mass of the monoisotopic peak in the individual isotopic distribution, and for space reasons, $\mathrm{M}+\mathrm{Li}$ is abbreviated to $\mathrm{M}$.

a ceramide part containing two hydrophobic tails. GSLs are expressed with heterogeneity within both the carbohydrate and ceramide components, and multistage dissociation is often necessary to obtain complete structural information for each portion of the glycolipid. Figure 2 shows the SORI-CID mass spectra from a GSL obtained from pig intestine. As in the previous example $\Delta \nu$ was set to $1.5 \%$ of $\nu_{+}$, while the irradiation amplitude was held constant at $10 \mathrm{~V}_{\mathrm{bp}}$. Under these conditions, SORI-CID of the lithiated molecular ion $\mathrm{m} / \mathrm{z}$ 824.68 generated a number of primary fragment ions over a broad $\mathrm{m} / \mathrm{z}$ range, accompanied by low abundance secondary fragments (Figure 2a). The observed fragmentation pattern is characteristic of GSLs containing an $\alpha$-hydroxy fatty acyl (FA) chain, indicated by the presence of an O-ion [34], and trihydroxysphinganine as the long chain base (LCB), indicated by a $\mathrm{Y} /[\mathrm{C}, \mathrm{N}]-$ ion [35]. To confirm this assignment and to obtain further structural information, a number of primary fragment ions were subjected to SORI-CID, using the same SORI settings $\left(\Delta \nu=1.5 \% \nu_{+}, 10 \mathrm{~V}_{\mathrm{bp}}\right.$, Figure $2 \mathrm{~b}-\mathrm{d}$ ). In this experiment, as $\Delta \nu$ was varied from 1950 to $5520 \mathrm{~Hz}$, the optimal SORI amplitude remained constant at $10 \mathrm{~V}_{\mathrm{bp}}$. In contrast to the oligosacharide example shown in Figure 1, the GSLs consist of two parts with structurally and chemically different characteristics. Despite this difference, no optimization of the irradiation amplitude was necessary when $\Delta \nu$ was calculated as a percentage of $\nu_{+}$. The obtained data were consistent with the expected structure for the glycolipid: A galactosylceramide having t18:0 as the LCB and h22:0 as the FA chain (see scheme in Figure 2).

In contrast to branched oligosaccharides and glycolipids, non-modified polypeptides have linear structures, and therefore often do not require the multiple dissociation stages. Nevertheless, since polypeptides are observed primarily as singly-charged ions in MALDI, the $m / z$ values of the ions selected for SORICID analysis may vary over a broad range and therefore, simplification of SORI-CID optimization would also aid their routine analysis. Using the same settings as in the previous examples, SORI-CID was performed on a number of peptides in the $\mathrm{m} / \mathrm{z}$ range $500-3500$ (Figure 3). For SORI-CID of each peptide, $\Delta \nu=1.5 \% \nu_{+}$ and an irradiation amplitude of $10 \mathrm{~V}_{\mathrm{bp}}$ was used. As was the case for the oligosaccharide and glycoconjugate samples, no precursor ions were observed in the $\mathrm{MS}^{2}$ spectra, indicating complete dissociation, but the overall fragmentation pattern varied widely among the studied peptides. This was expected, because the fragmentation pathways of singly charged peptides are strongly dependent on their amino acid composition [36]. Fibrinopeptide A (Figure 3c), for example, was cleaved only at the acidic residues, a pattern that is commonly observed for CID of C-terminal Arg-containing peptides [37]. Other peptides (Figure $3 b$ and $d$ ) exhibited more complete backbone fragmentation as well as abundant losses of water and ammonia. For some peptides, only abundant losses of water and/or ammonia were observed, without any polypeptidechain fragmentation (data not shown). For these, further increase of the irradiation amplitude resulted in some secondary fragmentation products, corresponding to internal fragment ions and additional water and/or ammonia losses. Thus, although the precalculated SORI parameters provided good starting conditions for peptides, tuning of the irradiation amplitude for certain peptides was required to enhance secondary fragmentation.

\section{Discussion}

SORI-CID is a general method for collisionally activating ions trapped in an FTMS cell and is frequently used for structural analysis of various biopolymers. In order to achieve the desired fragmentation level, both the irradiation frequency and amplitude are usually opti- 


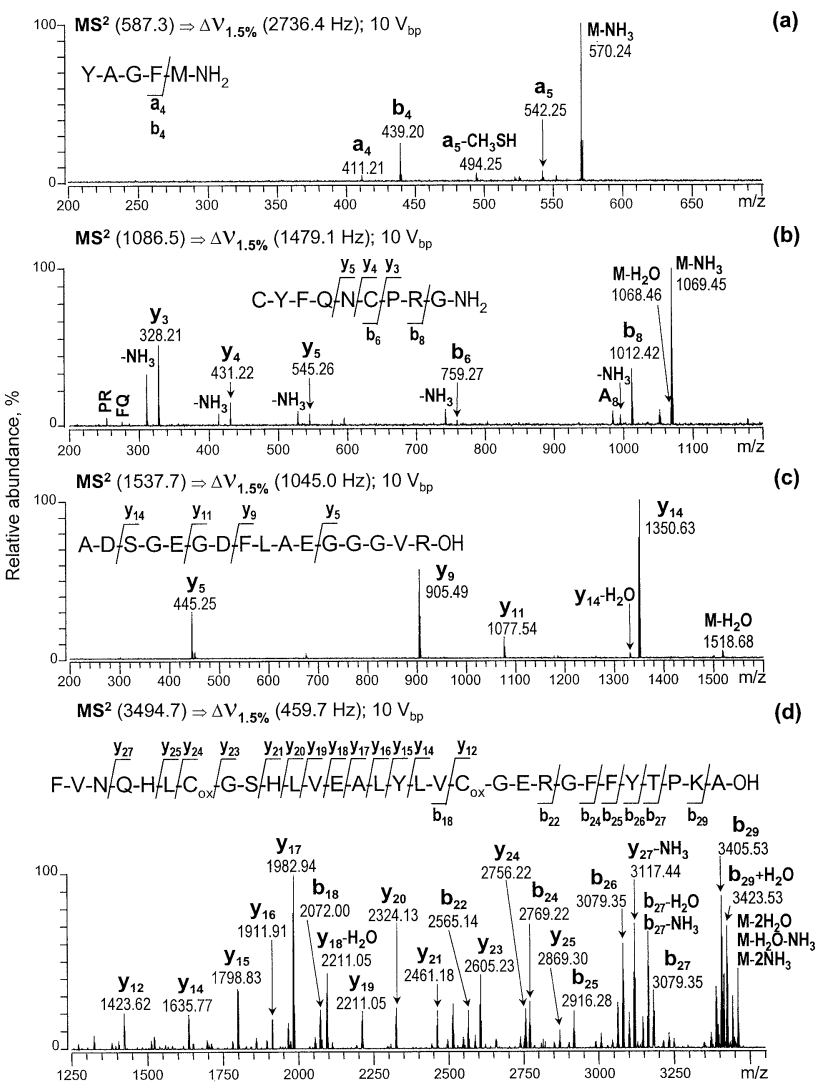

Figure 3. MALDI FT SORI-CID mass spectra of four different singly-charged protonated peptides using $\Delta \nu=1.5 \% \quad \nu_{+}$: (a) D-Ala ${ }^{2}$ Met $^{5}$-enkephalinamide (YAGFM-NH $\left.{ }_{2}\right), m / z 587.3(\Delta \nu=$ $2736.4 \mathrm{~Hz}$ ); (b) reduced $\mathrm{Arg}^{8}$-vasopressin (CYFQNCPRG-OH), $m / z$ $1086.5(\Delta \nu=1479.1 \mathrm{~Hz})$; (c) fibrinopeptide A (ADSGEGDFLAEGGGVR-OH), m/z $1537.7(\Delta \nu=1045.0 \mathrm{~Hz})$; (d) oxidized insulin

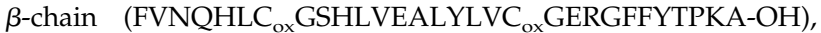
$m / z 3494.7(\Delta \nu=459.7 \mathrm{~Hz})$. Fragment ions are labeled according to standard peptide fragmentation nomenclature [40, 41]. All mass values represent the mass of the monoisotopic peak in the individual isotopic distribution, and for space reasons, $\mathrm{M}+\mathrm{H}$ is abbreviated to $\mathrm{M}$.

mized for each selected precursor ion, and SORI-CID experimental conditions reported in the literature therefore vary considerably. The requirement for extensive optimization of the SORI-CID parameters becomes particularly time consuming when the $\mathrm{m} / \mathrm{z}$ of the ions to be activated varies considerably during the analysis, which is especially the case for the singly charged ions generated by MALDI. When only a small sample amount is available, the optimization of the SORI parameters often limits the number of precursor ions that can be selected for fragmentation. The multiply charged ions generated by ESI typically fall within a narrower $\mathrm{m} / \mathrm{z}$ range, and thus the time required for optimization of the SORI parameters is usually shorter when selecting a new precursor ion. However, the precalculation of SORI-CID parameters described here should be useful when ESI-FTMS is used for on-line detection of analytes separated by liquid chromatography or capillary electrophoresis, for which the available analysis time for each sample component is limited by the analyte elution time window.

As was demonstrated experimentally with examples of various biopolymers, the described approach for calculating $\Delta \nu$ had the result that the optimal amplitude was significantly less dependent on the $\mathrm{m} / \mathrm{z}$ value of the precursor ion. The rationale for calculating $\Delta \nu$ as a constant percentage of $\nu_{+}$is outlined in the following: The average kinetic energy of an ion in the laboratory frame obtained during SORI irradiation described by the following equation [8]:

$$
\left\langle E_{l a b}\right\rangle=\frac{q E_{0}^{2}}{8 \pi B} \frac{\nu_{+}}{\Delta \nu^{2}}
$$

where $B$ is the magnetic field; $E_{0}$ is the amplitude of the excitation electric field; $q$ is the charge of the ion; $\nu_{+}$is the reduced cyclotron frequency of the ion, and $\Delta \nu$ is the SORI frequency offset. With $\Delta \nu=\mathrm{k} \times \nu_{+}(\mathrm{k}$ is a constant) and taking the approximation $\nu_{+} \approx \nu_{\mathrm{c}}=$ $q B / 2 \pi m,\left\langle E_{\text {lab }}\right\rangle$ is directly proportional to the mass of the ion:

$$
\left\langle E_{\text {lab }}\right\rangle=\frac{q E_{0}^{2}}{8 \pi B} \frac{1}{k^{2} \nu_{+}} \cong \frac{m E_{0}^{2}}{4 B^{2} \times k^{2}}
$$

Consequently, for large molecular ions with molecular mass $(m)$ significantly larger than the molecular mass of the collision gas $(M)$, the center-of-mass collision energy $\left(\left\langle E_{\text {com }}\right\rangle\right)$ is roughly independent of the mass of the ion:

$$
\left\langle E_{\text {com }}\right\rangle=\frac{M}{M+m}\left\langle E_{\text {lab }}\right\rangle \cong \frac{M}{m}\left\langle E_{\text {lab }}\right\rangle=\frac{M E_{0}^{2}}{4 B^{2} \times k^{2}}
$$

Thus when $\Delta \nu=\mathrm{k} \times \nu_{+}$, according to Eq 3, $\left\langle E_{c o m}\right\rangle$ is independent of the $\mathrm{m} / \mathrm{z}$ of the ion selected for fragmentation.

In all experiments described here, $\mathrm{k}=0.015(1.5 \%)$ was empirically found to be an optimal value. This value represents a balance between high fragmentation efficiency and the need for a high degree of control of the fragment ion population produced. For example, the calculation of the SORI frequency offset as $\Delta \nu=$ $1.0 \% \nu_{+}$caused more abrupt changes in the observed fragmentation pattern upon small amplitude changes, as expected. In our FTMS system, for collision gas pressure in the analyzer region raised transiently to $10^{-6}$ torr region and the calculation $\Delta \nu=0.015 \nu_{+}$, the optimal SORI amplitude varied between $8-18 \mathrm{~V}_{\mathrm{bp}}$ depending on the desired fragmentation degree. For all analyzed compounds and independent of the $\mathrm{m} / \mathrm{z}$ of the precursor ion, the amplitude of $10 \mathrm{~V}_{\mathrm{bp}}$ resulted in complete dissociation of selected precursor ions, with formation mainly of primary fragment ions. In cases when the observed fragment ions were not sufficient for structural characterization, the amplitude was increased to enhance secondary fragment ion formation 
or to influence particular fragment ion abundance. Thus for the amplitude range of 8-18 $V_{b p}$, the fragmentation pattern varied from formation of only primary fragment ions with only partial dissociation of the precursor ion (8-9 $\left.V_{b p}\right)$, to formation of mainly secondary fragment ions $\left(18 \mathrm{~V}_{\mathrm{bp}}\right)$ with typical steps of the amplitude changes of $2 \mathrm{~V}_{\mathrm{bp}}$ to control selected fragment ion intensities.

For the multiple stage dissociation experiments frequently required for oligosaccharide and glycoconjugate samples, another advantage of calculating $\Delta \nu$ as a percentage of $\nu_{+}$became apparent: The single parameter to be optimized was the SORI amplitude, and adjustment was required only for the first dissociation stage. For example, as was shown in the SORI-CID analysis of the glycolipid sample, the SORI frequency offset changed from $1900 \mathrm{~Hz}$ to $5500 \mathrm{~Hz}$, while the amplitude of the SORI pulse remained constant for all the analyzed ions. These observations were consistent with our results obtained for a variety of carbohydrates and glycolipid mixtures, with components varying in their $\mathrm{m} / \mathrm{z}$ values by a few units to several hundreds.

\section{Conclusions}

A general method of precalculating parameters for SORI-CID is demonstrated for singly charged MALDIgenerated ions over a 10-fold range of masses (and frequencies). This method considerably simplified optimization of SORI-CID for analysis of carbohydrates, glycoconjugates, and peptides, requiring optimization of only a single experimental parameter (the irradiation amplitude) and only for the first MS/MS stage. Furthermore, the default amplitude used $\left(10 \mathrm{~V}_{\mathrm{bp}}\right)$ was a good initial value for fragmentation of all molecular types studied. The optimal amplitude varied slightly with the lability of the molecule, but was independent of $\mathrm{m} / \mathrm{z}$ in the range of 300-3500. The described approach for calculating the SORI frequency offset should be applicable to all FTMS instruments, but the irradiation amplitude $\left(E_{0}\right)$ should be adjusted, according to $\mathrm{Eq} 3$ to take into accounts variation in the magnetic field strength and/or cell geometry.

\section{Acknowledgments}

The carbohydrate sample was provided by B. N. Singh (SUNY Health Science Center, Syracuse, NY) as part of an on-going collaboration and the glycolipid sample was a gift from S. Teneberg and K.-A. Karlsson (Göteborg University, Sweden). This research was supported by NIH grant P41 RR10888.

\section{References}

1. Comisarow, M. B.; Marshall, A. G. Frequency-Sweep Fourier Transform Ion Cyclotron Resonance Spectroscopy. Chem. Phys. Lett. 1974, 26, 489-490.

2. Amster, I. J. Fourier Transform Mass Spectrometry. J. Mass Spectrom. 1996, 31, 1325-1337.
3. Marshall, A. G.; Hendrickson, C. L.; Jackson, G. S. Fourier Transform Ion Cyclotron Resonance Mass Spectrometry. Mass Spectrom. Rev. 1998, 17, 1-35.

4. Hillenkamp, F.; Karas, M.; Beavis, R. C.; Chait, B. T. MatrixAssisted Laser Desorption/Ionization Mass Spectrometry of Biopolymers. Anal. Chem. 1991, 63, 1193A-1202A.

5. Gauthier, J. W.; Trautman, T. R.; Jaconson, D. B. Sustained Off-Resonance Irradiation for Collision-Activated Dissociation Involving Fourier Transform Mass Spectrometry. CollisionActivated Dissociation Technique that Emulates Infrared Multiphoton Dissociation. Anal. Chim. Acta 1991, 246, 211-225.

6. Guan, S.; Marshall, A. G.; Wahl, M. C. MS/MS with High Detection Efficiency and Mass Resolving Power for Product Ions in Fourier Transform Ion Cyclotron Resonance Mass Spectrometry. Anal. Chem. 1994, 66, 1363-1367.

7. Hofstadler, S. A.; Wahl, J. H.; Bakhtiar, R.; Ander, G. A.; Bruce, J. E.; Smith, R. D. Capillary Electrophoresis Fourier Transform Ion Cyclotron Resonance Mass Spectrometry with Sustained Off-Resonance Irradiation for the Characterization of Protein and Peptide Mixtures. J. Am. Soc. Spectrom. 1994, 5, 894-899.

8. Huang, Y.; Pasa-Tolic, L.; Guan, S.; Marshall, A. G. CollisionInduced Dissociation for Mass Spectrometric Analysis of Biopolymers: High-Resolution Fourier Transform Ion Cyclotron Resonance MS ${ }^{4}$. Anal. Chem. 1994, 66, 4385-4389.

9. Senko, M. W.; Speir, J. P.; McLafferty, F. W. Collisional Activation of Large Multiply Charged Ions Using Fourier Transform Mass Spectrometry. Anal. Chem. 1994, 66, 28012808.

10. Schwartz, B. L.; Bruce, J. E.; Anderson, G. A.; Hofstadler, S. A.; Rockwood, A. L.; Smith, R. D.; Chilkoti, A.; Stayton, P. S. Dissociation of Tetrameric Ions of Noncovalent Streptavidin Complexes Formed by Electrospray Ionization. J. Am. Soc. Mass Spectrom. 1995, 6, 459-465.

11. Wu, Q. Y.; Vanorden, S.; Cheng, X. H.; Bakhtiar, R.; Smith, R. D. Characterization of Cytochrome C Variants with HighResolution FTICR Mass Spectrometry-Correlation of Fragmentation and Structure. Anal. Chem. 1995, 67, 2498-2509.

12. Little, D. P.; Aaserud, D. J.; Valaskovic, G. A.; McLafferty, F. W. Sequence Information from 42-108-Mer DNAs (complete for a 50-Mer) by Tandem Mass Spectrometry. J. Am. Chem. Soc. 1996, 118, 9352-9359.

13. Solouki, T.; Pasa-Tolic, L.; Jackson, G. S.; Guan, S.; Marshall, A. G. High-Resolution Multistage MS, $\mathrm{MS}^{2}$, and $\mathrm{MS}^{3}$ MatrixAssisted Laser Desorption/Ionization FT-ICR Mass Spectra of Peptides from a Single Laser Shot. Anal. Chem. 1996, 68, 3718-3725.

14. Heck, A. J. R.; Derrick, P. J. Ultrahigh Mass Accuracy in Isotope-Selective Collision-Induced Dissociation Using Correlated Sweep Excitation and Sustained Off-Resonance Irradiation-A Fourier Transform Ion Cycltron Resonance Mass Spectrometry Case Study on the $[\mathrm{M}+2 \mathrm{H}]^{(2+)}$ Ion of Bradykinin. Anal. Chem. 1997, 69, 3603-3607.

15. Kelleher, N. L.; Nicewonger, R. B.; Begley, T. P.; McLafferty, F. W. Identification of Modification Sites in Large Biomolecules by Stable Isotope Labeling and Tandem High Resolution Mass Spectrometry-The Active Site Nucleophile of Thiaminase I. J. Biol. Chem. 1997, 272, 32215-32220.

16. Kelleher, N. L.; Taylor, S. V.; Grannis, D.; Kinsland, C.; Chiu, H. J.; Begley, T. P.; McLafferty, F. W. Efficient Sequence Analysis of the Six Gene Products $(7-74 \mathrm{kDa})$ from the Escherichia coli Thiamin Biosynthetic Operon by Tandem HighResolution Mass Spectrometry. Protein Science 1998, 7, 17961801.

17. Lavanant, H.; Derrick, P. J.; Heck, A. J.; Mellon, F. A. Analysis of Nisin A and Some of Its Variants Using Fourier Transform Ion Cyclotron Resonance Mass Spectrometry. Anal. Biochem. 1998, 255, 74-89. 
18. Kelleher, N. L.; Lin, H. Y.; Valaskovic, G. A.; Aaserud, D. J.; Fridriksson, E. K.; McLafferty, F. W. Top Down Versus Bottom Up Protein Characterization by Tandem High-Resolution Mass Spectrometry. J. Am. Chem. Soc. 1999, 121, 806-812.

19. Maier, C. S.; Yan, X.; Harder, M. E.; Schimerlik, M. I.; Deinzer, M. L.; Pasa-Tolic, L.; Smith, R. D. Electrospray Ionization Fourier Transform Ion Cyclotron Resonance Mass Spectrometric Analysis of the Recombinant Human Macrophage Colony Stimulating Factor Beta and Derivatives. J. Am. Soc. Mass Spectrom. 2000, 11, 237-243.

20. Cancilla, M. T.; Penn, S. G.; Lebrilla, C. B. Alkaline Degradation of Oligosaccharides Coupled with Matrix-Assisted Laser Desorption/Ionization Fourier Transform Mass Spectrometry: A Method for Sequencing Oligosaccharides. Anal. Chem. 1998, $70,663-672$.

21. Solouki, T.; Reinhold, B. B.; Costello, C. E.; O'Malley, M.; Guan, S.; Marshall, A. G. Electrospray Ionization and MatrixAssisted Laser Desorption/Ionization Fourier Transform Ion Cyclotron Resonance Mass Spectrometry of Permethylated Oligosaccharides. Anal. Chem. 1998, 70, 857-864.

22. Cancilla, M. T.; Wong, A. W.; Voss, L. R.; Lebrilla, C. B. Fragmentation Reactions in the Mass Spectrometry Analysis of Neutral Oligosaccharides. Anal. Chem. 1999, 71, 3206-3218.

23. Gaucher, S. P.; Cancilla, M. T.; Phillips, N. J.; Gibson, B. W.; Leary, J. A. Mass Spectral Characterization of Lipooligosaccharides from Haemophilus influenzae 2019. Biochemistry 2000, 39, 12406-12414.

24. Penn, S. G.; Cancilla, M. T.; Lebrilla, C. B. Fragmentation Behavior of Multiple-Metal-Coordinated Acidic Oligosaccharides Studied by Matrix-Assisted Laser Desorption/Ionization Fourier Transform Mass Spectrometry. Int. J. Mass Spectrom. 2000, 196, 259-269.

25. Hettich, R. L.; Stemmler, E. A. Investigation of Oligonucleotide Fragmentation with Matrix-Assisted Laser Desorption/ Ionization Fourier Transform Mass Spectrometry and Sustained Off-Resonance Irradiation. Rapid Commun. Mass Spectrom. 1996, 10, 321-327.

26. Flora, J. W.; Hannis, J. C.; Muddiman, D. C. High-Mass Accuracy of Product Ions Produced by SORI-CID Using a Dual Electrospray Ionization Source Coupled with FTICR Mass Spectrometry. Anal. Chem. 2001, 73, 1247-1251.

27. Pastor, S. J.; Wilkins, C. L. Sustained Off-Resonance Irradiation and Collision-Induced Dissociation for Structural Analysis of Polymers by MALDI-FTMS. Int. J. Mass Spectrom. Ion Processes 1998, 175, 81-92.

28. Laskin, J.; Byrd, M.; Futrell, J. Internal Energy Distributions Resulting from Sustained Off-Resonance Excitation in FTMS. I. Fragmentation of the Bromobenzene Radical Cation. Int. J. Mass Spectrom. 2000, 196, 285-302.
29. Gorshkov, M. V.; Pasa-Tolic, L.; Smith, R. D. Pressure Limited Sustained Off-Resonance Irradiation for Collisionally-Activated Dissociation in Fourier Transform Mass Spectrometry. J. Am. Soc. Mass Spectrom. 1999, 10, 15-18.

30. Beu, S. C.; Laude, D. A. Open Trapped Ion Cell Geometries for Fourier Transform Ion Cyclotron Resonance Mass Spectrometry. Int. J. Mass Spectrom. Ion Processes 1992, 112, 215-230.

31. Guan, S. H.; Marshall, A. G. Stored Waveform Inverse Fourier Transform (SWIFT) Ion Excitation in Trapped-Ion Mass Spectrometry-Theory and Applications. Int. J. Mass Spectrom. Ion Processes 1996, 158, 5-37.

32. Cancilla, M. T.; Penn, S. G.; Carroll, J. A.; Lebrilla, C. B. Coordination of Alkali Metals to Oligosaccharides Dictates Fragmentation Behavior in Matrix-Assisted Laser Desorption Ionization Fourier Transform Mass Spectrometry. J. Am. Chem. Soc. 1996, 118, 6736-6745.

33. Penn, S. G.; Cancilla, M. T.; Green, M. K.; Lebrilla, C. B. Direct Comparison of Matrix-Assisted Laser Desorption/Ionization and Electrospray Ionization in the Analysis of Gangliosides by Fourier Transform Mass Spectrometry. Eur. Mass Spectrom. 1997, 3, 67-79.

34. Adams, J.; Ann, Q. H. Structure Determination of Sphingolipids by Mass Spectrometry. Mass Spectrom. Rev. 1993, 12, 51-85.

35. Mirgorodskaya, E.; Costello, C. E. Characterization of Neutral Glycosphingolipids by MALDI FTMS Using SORI-CID. Proceedings of the 48th ASMS Conference on Mass Spectrometry and Allied Topics; Long Beach CA, June, 2000, pp 1097-1098.

36. Qin, J.; Chait, B. T. Collision-Induced Dissociation of Singly Charged Peptide Ions in a Matrix-Assisted Laser Desorption Ionization Ion Trap Mass Spectrometer. Int. J. Mass Spectrom. 1999, 191, 313-320.

37. Tsaprailis, G.; Nair, H.; Somogyi, A.; Wysocki, V. H.; Zhong, W. Q.; Futrell, J. H.; Summerfield, S. G.; Gaskell, S. J. Influence of Secondary Structure on the Fragmentation of Protonated Peptides. J. Am. Chem. Soc. 1999, 121, 5142-5154.

38. Domon, B.; Costello, C. E. A Systematic Nomenclature for Carbohydrate Fragmentations in FAB-MS/MS Spectra of Glycoconjugates. Glycoconj. J. 1988, 5, 397-409.

39. Perreault, H.; Costello, C. E. Differentiation of Cerebroside Isomers and Study of Fragmentation by Liquid Secondary Ion Mass Spectrometry and Mass Spectrometry. Mass Spectrometry of Selected Derivatives. Can. J. Chem. 1996, 74, 1682-1695.

40. Roepstorff, P.; Fohlman, J. Proposal for a Common Nomenclature for Sequence Ions in Mass Spectra of Peptides. Biomed. Mass Spectrom. 1984, 11, 601.

41. Biemann, K. Contributions of Mass Spectrometry to Peptide and Protein Structure. Biomed. Environ. Mass Spectrom. 1988, $16,99-111$. 